\title{
Working with Gravitational-Wave sky localizations: new methods and implementations
}

\section{Giuseppe Greco*}

Università degli Studi di Urbino “Carlo Bo”, I-61029 Urbino, Italy

INFN, Sezione di Firenze, I-50019 Sesto Fiorentino, Firenze, Italy

E-mail: giuseppe.greco@uniurb.it

\section{Marica Branchesi}

Gran Sasso Science Institute (GSSI), I-67100 L'Aquila, Italy

INFN, Laboratori Nazionali del Gran Sasso, I-67100 Assergi, Italy

\section{Eric Chassande-Mottin}

APC, AstroParticule et Cosmologie, Université Paris Diderot, CNRS/IN2P3, CEA/Irfu,

Observatoire de Paris, Sorbonne Paris Cité, F-75205 Paris Cedex 13, France

\section{Michael W. Coughlin}

LIGO, California Institute of Technology, Pasadena, CA 91125, USA

\section{Giulia Stratta}

INAF - Osservatorio di Astrofisica e Scienza dello Spazio, Via Piero Gobetti 93/3, I-40129

Bologna, Italy and INFN, Sezione di Firenze, I-50019 Sesto Fiorentino, Firenze, Italy

\section{Gergely Dálya}

Institute of Physics, Eötvös University, 1117 Budapest, Hungary

MTA-ELTE Astrophysics Research Group, 1117 Budapest, Hungary

\section{Gary Hemming}

European Gravitational Observatory (EGO), I-56021 Cascina, Pisa, Italy

\section{Luca Rei}

INFN, Sezione di Genova, Via Dodecaneso 33, 16146 Genova, Italy

\section{Enzo Brocato}

INAF, Osservatorio Astronomico d'Abruzzo, Via Mentore Maggini Teramo, TE, I-64100, Italy

Pierre Fernique, Thomas Boch, Sébastien Derriere, Matthieu Baumann, Francoise Genova and Mark Allen

CDS, Université de Strasbourg, CNRS, Observatoire astronomique de Strasbourg, UMR 7550,

11 rue de l'Université, 67000 Strasbourg, France 
The era of multi-messenger astrophysics with Gravitational Waves $(\mathrm{GW})$ requires the exploration and development of suitable methods and tools for real-time analysis as well as post-processing activities. The irregular and complex shapes of the GW sky localizations represent a new challenge for observational astronomers, who need to work with fast tiling, catalog queries, transient localizations, visibility and sky map comparisons. Here we show how gravitational-wave sky maps can be easily and efficiently visualized and processed using Multi-Order Coverage (MOC) maps. These maps are based on HEALPix sky tessellation which uses both Python language and the recent implementation in Aladin Desktop/Lite. In addition to this, we describe a specific interactive script, named GWsky, that we developed to effectively tile the sky localization of a gravitational-wave event providing accurate telescope pointings. We also show applications of these methods and tools for educational purposes in Virtual Reality Apps, high resolution images, and basic sonification of the GW sky maps.

Finally, we describe possible evolutions of such implementations when three or more groundbased interferometers will be involved in a gravitational-wave source localization (i.e. Virgo, LIGO-Hanford, LIGO-Livingston, KAGRA, LIGO-India) with a corresponding increase of the sky map resolution.

The New Era of Multi-Messenger Astrophysics - Asterics2019

25 - 29 March, 2019

Groningen, The Netherlands

* Speaker. 


\section{MOC and GW sky localizations}

In this section, we describe the Multi-Order Coverage map method and the basic algorithm to calculate a credible region of a gravitational-wave sky localization. Afterwards, we show how the credible region (confidence level) can easily be decoded in a MOC map.

\subsection{MOC: Multi-Order Coverage map}

The gravitational-wave sky localizations are given as all-sky images stored in the HEALPix ${ }^{1}$ projection, and they are typically irregularly shaped. Only for particularly accurately localized events, the sky localization region can be well described by an ellipse ${ }^{2}$. Taking advantage of the HEALPix projection format, we develop a procedure to apply the MOC method to identify the credible regions of the GW sky localization. MOC stands for Multi-Order Coverage map. It is a standard of the Virtual Observatory ${ }^{3}$ which provides a multi-scale mapping based on HEALPix sky tessellation. Basically, the algorithm maps irregular and complex sky regions into hierarchically grouped predefined cells. Each MOC cell is defined by two numbers: the hierarchy level (HEALPix ORDER) and the pixel index (HEALPix IPIX). The NUNIQ encoding scheme defines an algorithm for packing an (ORDER, IPIX) pair into a single integer for compactness [1]. More details about the HEALPix Unique Identifier and NUNIQ schemes are in the HEALPix Primer ${ }^{4}$. MOCs are serialized as FITS or JSON files. The MOC resolution is determined by the map resolution parameter $N_{\text {side }}$, which is used for defining the resolution of the grid. For a typical $N_{\text {side }}=512$, the MOC order resolution is order $=9 ;\left(N_{\text {side }}=2^{\text {order }}\right)$.

The MOC maps make database queries for retrieving objects and logical operations (such as union, intersection, subtraction, difference) extremely simple and fast (generally a few seconds) even for very complex sky regions. If databases are adapted to support MOC based queries, such as VizieR ${ }^{5}$, they offer a useful method allowing any support of sky region query.

\subsection{Credible region of a gravitational-wave sky localization}

The contours of a GW sky localization, which enclose a given percentage of the total probability, are constructed using a water-filling algorithm: the pixels from most probable to least are ranked, and summed up to get a fixed level of probability [2]. The enclosed area within a given probability level contour of a GW sky map can be effectively described through the Multi-Order Coverage (MOC) method. In practice, the HEALPix pixels inside a given contour plot are extracted, and, the MOC coverage is generated from the table made up from the pixels. Every single level of probability can be used as a regular MOC.

\section{Aladin Desktop}

The Graphical User Interface (GUI) of the Aladin Desktop [3] implements the code described in Section 1. The Aladin version $\mathbf{v 1 0 . 0 7 6}$ is considered here.

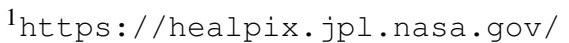

${ }^{2}$ https://emfollow.docs.ligo.org/userguide

${ }^{3}$ http: //www.ivoa. net/

${ }^{4}$ https://healpix.sourceforge.io/pdf/intro.pdf

5 http://vizier.u-strasbg.fr/viz-bin/VizieR
} 
The same tasks can be performed with a dedicated Python module named mocpy ${ }^{6}$. In particular the method MOC.fill() and MOC.border() support the matplotlib graphics library for highquality publication plots. The first methods draws the interior of a MOC map and the second one its perimeter.

\subsection{Main GUI commands}

The sequence of the Aladin GUI commands to create a credible region at a defined confidence level is reported below.

From the main menu press $\rightarrow$ Coverage $\rightarrow$ Generate a MOC based on..$\rightarrow$ The current probability skymap $\rightarrow$ MOC generation window

The associated Properties windows allow to change the drawing methods in perimeter in order to simultaneously visualize multiple confidence levels. This operation facilitates tiling operations by telescopes monitoring the highest probability areas - see Section 3 . The enclosed sky area in square degrees and the percentage of the sky coverage are quoted for each MOC either $i$ ) by leaving the cursor on the corresponding plan loaded in the Aladin stack or $i i$ ) by opening the associated Properties windows.

\subsection{Aladin data collections tree and 3D sky maps}

Singer et al. (2016) [4] discuss a fast algorithm for obtaining three-dimensional probability estimates of sky location and luminosity distance from observations of binary compact object mergers with Advanced LIGO and Virgo. Combining the reconstructed gravitational-wave volumes with positions and redshifts of possible host galaxies provides a manageable list of sky location targets to search for the electromagnetic counterpart of the gravitational-wave signal. These tasks can efficiently be performed in the Aladin Desktop using the data collections tree ${ }^{7}$ and the filter methods ${ }^{8}$ as follows.

Aladin data collections tree $\rightarrow$ Select $\rightarrow$ click on the catalog item

$\rightarrow$ in the popup menu check $\rightarrow$ by region \& MOC

Catalog $\rightarrow$ Create a filter $\rightarrow$ Properties window $\rightarrow$ Advanced mode

$\rightarrow$ or enter your filter definition:

An example about the Aladin filter using as galaxy selection the marginal distance posterior distribution integrated over the whole sky is reported below:

$\$\{$ Dist $\}>$ DISTMEAN-DISTSTB \&\& $\$\{$ Dist $\}<$ DISTMEAN+DISTSTB $\{$ draw

The posterior mean distance $(\mathrm{Mpc})$ and the posterior standard deviation of distance $(\mathrm{Mpc})$ are reported in the fits file header with the keywords DISTMEAN and DISTSTB.

\section{GWsky tool: tiling the sky map}

Various strategies to cover the gravitational-wave sky-localizations have been proposed in order to increase the chances of capturing an electromagnetic counterpart [5] and [6] - to name a few.

\footnotetext{
${ }^{6}$ https://pypi.org/project/MOCPy/

${ }^{7}$ https: / / www youtube.com/watch?v=IG_6Eh9EKKk

${ }^{8}$ http: //cdsweb.u-strasbg.fr/boch/doc/filters.htx
} 
In this context, we develop a tool named GWsky ${ }^{9}$ which is a Python script devoted to run a sequence of telescope pointings across the sky localization of gravitational-wave signals. A simple GUI supports the users to interactively tile the gravitational-wave sky map taking into account information for each assigned telescope pointing, such as airmass plot, integrated probability and the conditional distance distribution for the 3D sky map, catalog query table. A semi-transparent background is applied to the movable windows. This graph attribute combined with the reduced window size makes easy to work in the overlay mode between the different Aladin planes. GWsky interacts with the Aladin Desktop via SAMP (Simple Application Messaging Protocol). SAMP ${ }^{10}$ is the Virtual Observatory messaging protocol that enables astronomy software to interoperate and communicate [16]. It is used by a number of other tools such as TOPCAT ${ }^{11}, \mathrm{SAO}_{\mathrm{DS}} 9^{12}$, and Aladin, thus, it is possible to send and receive data to and from these tools.

\section{Virtual Reality and Aladin Lite for inclusive education}

The HiPS (Hierarchical Progressive Surveys) [7] technology provides an efficient approach to generate high resolution images as well as Virtual Reality (VR) applications ${ }^{13,14}$.

HiPS can be visualized in Aladin Lite [8]. Aladin Lite is a lightweight version of the Aladin Desktop tool, running in the browser and geared towards simple visualization of a sky region. It is powered by the HTML5 canvas technology, currently supported by any modern browser. Aladin Lite successfully supports the MOC regions as separated layers. The main application was developed for the announcement of the first triple detection of GW170814 with the two LIGO interferometers and Virgo [9]. The interactive detection page also shows the main events issued to the astronomers during the first and second observational LIGO and Virgo runs and the sky localizations of GW170817/GRB170817/AT2019gfo [10] in light and gravity. We are working on the development of Aladin Lite for inclusive education. In more details, a specific chord is played when the cursor enters or leaves the MOC coverage. An audio file is added to explain the nature of each event and the main properties. The user can modify the frequency range or exclude the sound system with a check button. The frequency is mapped to the galactic latitude of the mouse cursor location with a stereo spatialization (left/right speaker) for the galactic longitude. An automatic tour is added using the Aladin Lite plugin Tour Navigator ${ }^{15}$ developed by T. Civera (CEFCA).

\section{Future developments}

The resolution of a GW sky localization with three or more detectors can reach a resolution considerably large $\left(N_{\text {side }}=2048\right)$. To mitigate the computational time, the Aladin Beta v10.117 integrates a more powerful algorithm to extract MOC from an HEALPix map. The HEALPix is converted in HiPS (for splitting the data in tiles) before applying the MOC method.

\footnotetext{
${ }^{9}$ https://github.com/ggreco77/GWsky

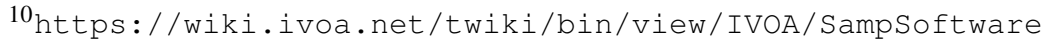

11 http://www.star.bris.ac.uk/ mbt/topcat/

12 http://ds9.si.edu/site/Home.html

${ }^{13}$ https://play.google.com/store/search?q=gwsky,

14 https://poly.google.com/user/dAGmDoi6BvB

15 http://www.cefca.es/cefca_en/tour_navigator_library
} 
A second approach is represented by the multiorder sky maps ${ }^{16}$. It is a new variant of the HEALPix format that is designed to overcome limitations in well-localized events and future gravitational-wave facilities. It uses HEALPix explicit indexing and the NUNIQ numbering scheme, which is closely related to multi-order coverage (MOC) maps in Aladin.

\section{References}

[1] P. Fernique, T. Boch, T. Donaldson, D. Durand, W. O'Mullane, M. Reinecke et al., "MOC - HEALPix Multi-Order Coverage map Version 1.0.” IVOA Recommendation 02 June 2014, June, 2014.

[2] L. P. Singer, L. R. Price, B. Farr, A. L. Urban, C. Pankow, S. Vitale et al., The First Two Years of Electromagnetic Follow-up with Advanced LIGO and Virgo, The Astrophysical Journal 795 (2014) 105 [1404.5623].

[3] F. Bonnarel, P. Fernique, O. Bienaymé, D. Egret, F. Genova, M. Louys et al., The ALADIN interactive sky atlas. A reference tool for identification of astronomical sources, Astronomy and Astrophysics, Supplement 143 (2000) 33.

[4] L. P. Singer, H.-Y. Chen, D. E. Holz, W. M. Farr, L. R. Price, V. Raymond et al., Going the Distance: Mapping Host Galaxies of LIGO and Virgo Sources in Three Dimensions Using Local Cosmography and Targeted Follow-up, The Astrophysical Journal, Letters 829 (2016) L15 [1603. 07333].

[5] S. Ghosh, S. Bloemen, G. Nelemans, P. J. Groot and L. R. Price, Tiling strategies for optical follow-up of gravitational-wave triggers by telescopes with a wide field of view, Astronomy and Astrophysics 592 (2016) A82 [1511.02673].

[6] M. W. Coughlin, D. Tao, M. L. Chan, D. Chatterjee, N. Christensen, S. Ghosh et al., Optimizing searches for electromagnetic counterparts of gravitational wave triggers, Monthly Notices of the Royal Astronomical Society 478 (2018) 692 [1803.02255].

[7] P. Fernique, M. G. Allen, T. Boch, A. Oberto, F.-X. Pineau, D. Durand et al., Hierarchical progressive surveys. Multi-resolution HEALPix data structures for astronomical images, catalogues, and 3-dimensional data cubes, Astronomy \& Astrophysics 578 (2015) A114 [1505. 02291].

[8] T. Boch and P. Fernique, Aladin Lite: Embed your Sky in the Browser, in Astronomical Data Analysis Software and Systems XXIII, N. Manset and P. Forshay, eds., vol. 485 of Astronomical Society of the Pacific Conference Series, p. 277, May, 2014.

[9] B. P. Abbott, R. Abbott, T. D. Abbott, F. Acernese, K. Ackley, C. Adams et al., GW170814: A Three-Detector Observation of Gravitational Waves from a Binary Black Hole Coalescence, Physical Review Letters 119 (2017) 141101 [1709.09660].

[10] B. P. Abbott, R. Abbott, T. D. Abbott, S. Abraham, F. Acernese, K. Ackley et al., Low-latency Gravitational-wave Alerts for Multimessenger Astronomy during the Second Advanced LIGO and Virgo Observing Run, The Astrophysical Journal 875 (2019) 161.

\section{Acknowledgements}

The authors acknowledge support from ASTERICS, a project funded by the European Commission under the Horizon2020 programme (id 653477), in the framework of ASTERICS Work Package 4 Data Access, Discovery and Interoperability.

\footnotetext{
16https://emfollow.docs.ligo.org/userguide/tutorial/multiorder_skymaps.html
} 